\title{
19. Tag der gesunden Ernährung am 7. März 2016
}

\section{Onkologie - Krebsprävention mit Messer und Gabel}

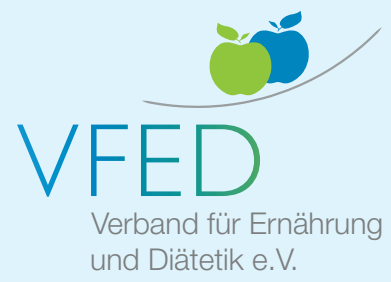

Bereits zum 19. Mal veranstaltet der Verband für Ernährung und Diätetik e.V. (VFED) den „Tag der gesunden Ernährung“. Mit diesem Aktionstag soll die Bevölkerung in Deutschland sowie in den deutschsprachigen Nachbarländern auf die Wichtigkeit der gesunden Ernährung aufmerksam gemacht werden. Der Tag der gesunden Ernährung wurde 1996 initiiert und wird vom VFED koordiniert. Jährlich finden so im gesamten deutschsprachigen Raum mehr als 2000 Aktionen zum Tag der gesunden Ernährung statt.

Der 19.Tag der gesunden Ernährung am 7. März 2016 fokussiert das Thema Prävention in der Onkologie. Jährlich wird bei etwa 500000 Menschen in Deutschland Krebs diagnostiziert, etwa die Hälfte aller Erkrankten stirbt. Heute können zwar mehr Erkrankte auf eine dauerhafte Heilung hoffen als noch vor 30 Jahren, jedoch gelten bösartige Tumorleiden laut dem statistischen Bundesamt als zweithäufigste Todesursache nach den HerzKreislauf-Erkrankungen.

Die Diagnose „Krebs“ ist für die meisten Betroffenen zunächst mit großer Unsicherheit und Ängsten verbunden. Besonders Gedanken an mögliche Therapienebenwirkungen, Schmerzen, Leiden und das Einbüßen von Lebensqualität kommen auf.

\section{Falsche Ernährung - ein erheblicher Risikofaktor}

Der Faktor Ernährung spielt sowohl bei der Entstehung als auch während und nach einer Tumortherapie eine wichtige Rolle. Das Deutsche Krebsforschungszentrum schätzt die Beteiligung von Ernährungsgewohnheiten an der Krebsentstehung auf $20-40 \%$. Somit wäre die Ernährung nach dem Konsum von Tabak

\section{Empfehlungen}

Auf Basis des WCRF (World Cancer Research Found)-Reports wurden 10 Empfehlungen zur Krebsprävention erstellt (Quelle: Prof. Dr. Claus Leitzmann [4]).

1. So schlank wie möglich bleiben.

2. Körperliche Aktivität sollte Teil des täglichen Lebens sein.

3. Der Verzehr energiedichter Lebensmittel sollte begrenzt werden.

4. Überwiegend pflanzliche Lebensmittel verzehren.

5. Den Verzehr von rotem Fleisch (Rind, Schwein, Schaf, Ziege) begrenzen. Den Verzehr von verarbeitetem Fleisch (gepökelt, gebeizt, geräuchert) vermeiden.

6. Begrenzung des Konsums alkoholischer Getränke.

7. Begrenzung des Salzkonsums. Der Verzehr von verschimmeltem Getreide oder Hülsenfrüchten ist zu vermeiden.

8. Der Nährstoffbedarf sollte ausschließlich durch Lebensmittel gedeckt werden.

9. Mütter sollten stillen; Säuglinge sollten gestillt werden.

10. Für ehemalige Krebspatienten gelten die Empfehlungen zur Krebsprävention.

der zweitgrößte Risikofaktor für die Entstehung von Krebs.

Eine gesunde Lebensweise mit einer ausgewogenen, nährstoffreichen Kost kann das Risiko einer Krebserkrankung senken, aber auch ungewollte Nebenwirkungen der Therapie mildern und sich positiv auf das allgemeine Wohlbefinden auswirken. Die Ernährungsberatung ist daher ein zentraler Aspekt sowohl in der Prävention als auch in der Krebstherapie ( $\vee$ Info).

Mit diesem Tag der gesunden Ernährung möchte der VFED die Bevölkerung auf die Bedeutung der präventiven Maßnahmen aufmerksam machen!

Nach Expertenmeinung kann etwa der Hälfte aller Neuerkrankungen vorgebeugt werden, wenn die Empfehlungen zur Krebsprävention in die eigene Lebensweise eingebunden werden.

Zahlreiche Faktoren im Bereich der Ernährung können das Krebsrisiko beeinflussen. Bestimmte Lebensmittelinhaltsstoffe, wie z.B. die Ballaststoffe, senken nachweislich das Risiko einiger Krebs- arten. Eine Vielzahl von Inhaltsstoffen wirkt antikanzerogen, beispielsweise Carotinoide oder Flavonoide. Hingegen gelten bestimmte Lebensmittel als risikoerhöhend, wie z.B. rotes Fleisch und/oder gepökeltes Fleisch.

\section{Prävention durch Beratung}

Nach derzeitiger Studienlage kann eine Ernährung bestehend aus vorwiegend pflanzlichen Lebensmitteln (Vollkornprodukten, frischem Obst und Gemüse), eingeschränktem Fleischkonsum, unter Vermeidung von energiedichten Nahrungsmitteln (Fast Food, gezuckerte Produkte) und mit begrenztem Alkoholkonsum zu einer deutlichen Minderung des Krebsrisikos beitragen. Eine Orientierung für eine ausgewogene Ernährungsweise bietet das VFED-Ernährungsdreieck ( Abb. 1).

Da Übergewicht und die Fettverteilung (viszerale Adipositas) mit einem erhöhten Krebsrisiko für bestimmte Krebs- 


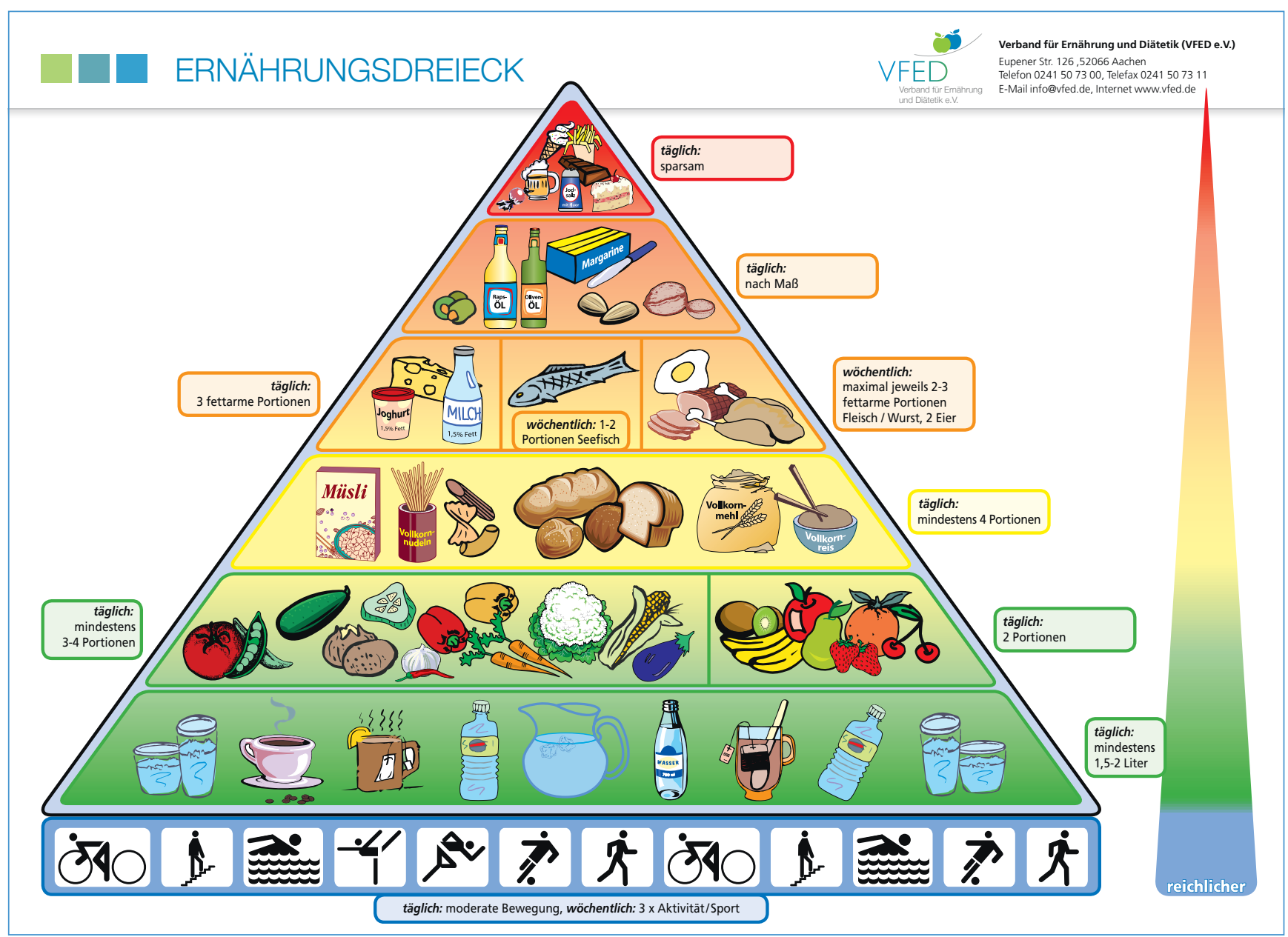

- Abb. 1 So gelingt die Beratung zur Krebsprävention: Das Ernährungsdreieck des VFED berücksichtigt die Prinzipien einer ausgewogenen Ernährung in Verbindung mit körperlicher Aktivität. (C) VFED e.V.

arten in Verbindung gebracht werden, ist nicht nur die Auswahl der Lebensmittel entscheidend, auch die Art der Zubereitung und die Energiebilanz sind maßgeblich von Bedeutung.

Mit dem Tag der gesunden Ernährung möchte der VFED Ernährungsfachkräfte dazu motivieren einen Aktionstag zu gestalten, um eine breite Bevölkerungsgruppe zu erreichen und das Bewusstsein für dieses wichtige Thema von besonderer Tragweite zu erhöhen. Ebenfalls kann dieser Aktionstag dazu genutzt werden, um neue Kontakte zu knüpfen und das berufliche Aufgabenfeld in der Bevölkerung bekannter zu machen.

\section{So können Ernährungs- fachkräfte mitmachen}

Nach persönlichen Möglichkeiten, Stärken und Vorlieben kann eine eigene Ver- anstaltung geplant werden. Potenzielle Aktionen sind z.B. ein Vortrag, Verkostungen, Interviews, Seminare, ein Kochkurs oder die Gestaltung eines Informationstisches. Durchführbar ist ein Aktionstag in einem Krankenhaus oder einer Klinik, einer Apotheke, einer Krankenkasse, einem Geschäft, der Bücherei, Volkshochschule oder anderen Bildungseinrichtungen.

Der eigenen Kreativität sind dabei keine Grenzen gesetzt. Jede(r) Interessierte kann seine Ideen umsetzen. Optional können Ernährungsfachkräfte eine Aktion allein oder gemeinsam mit Kolleginnen anbieten. Ebenfalls besteht die Möglichkeit, mit Kooperationspartnern (Firmen, Vereine) zusammen zu arbeiten, die das Vorhaben finanziell, mit Produkten oder Räumlichkeiten unterstützen. Obwohl der Tag der gesunden Ernährung auf den 7. März festgelegt ist, können die Aktionen auch einige Tage früher oder später stattfinden, ganz entsprechend den persönlichen Gegebenheiten.

Für weitere Anregungen sind auf der Internetseite des VFED unter http:// www.vfed.de/de/archiv_ Bilder der vorherigen Tage der gesunden Ernährung zu finden.

\section{So unterstützt Sie der VFED beim Aktionstag}

Für eine erfolgreiche Gestaltung der individuellen Veranstaltung zum 19.Tag der gesunden Ernährung stellt der VFED ein spezielles Aktionspaket (s.u.) zusammen, das in der Geschäftsstelle angefordert werden kann. Dieses enthält neben diversen Plakaten und Broschüren, ein zum Thema Krebsprävention erstelltes Leporello sowie ein Sonderheft „Onkologie“. Unter anderen sind Beiträge zu Entstehungsfaktoren, dem Ernährungs- 
zustand und der Stoffwechsellage von Tumorpatienten, Therapiemöglichkeiten, der ketogenen Ernährung und dem Umgang mit Therapienebenwirkungen vorgesehen.

Gesondert zum Aktionspaket wird eigens zum Thema ein Vortrag erstellt, der zusätzlich als Folienvortrag erworben werden kann.

Um die eigene Veranstaltung im Vorfeld bewerben zu können, erhält das Aktionspaket einen Leitfaden mit Tipps für die Presse- und Öffentlichkeitsarbeit. Überregional macht der VFED durch Presseartikel und Newsletter auf den Tag der gesunden Ernährung aufmerksam.

Wenn Sie am Tag der gesunden Ernährung teilnehmen möchten, melden Sie sich in der Geschäftsstelle des VFED. Sie finden zu diesem Zweck am Ende einen Rückmeldebogen, den Sie an die VFEDGeschäftsstelle zurückschicken können.

\section{Aktionspaket}

Folgende Inhalte sind geplant:

- 1 VFEDaktuell Fachmagazin „Onkologie“ (Neuauflage 2016, ca. 100 Seiten)

- 50 Leporellos zum Thema „Onkologie - Mit Schwerpunkt Prävention“

- 2 Plakate „Tag der gesunden Ernährung“ für die eigene Werbung

- 1 Broschüre „Gesund genießen bei erhöhten Cholesterin- und Triglyceridwerten“

- 1 Broschüre „Lecker und ausgewogen mit dem VFED-Ernährungsdreieck“

- 1 Plakat BMI für Erwachsene

- 1 Plakat BMI für Senioren

- 1 Plakat „Ernährungsdreieck“

- 1 Beratungsunterlage VFED Ess- und Aktivtagebuch

- 1 Poster Bewegungsprogramm für jedes Alter, DIN A4

- 10 Aufkleber „Ernährungsdreieck des VFED“

- 1 VFED-Kugelschreiber
- Bestellmöglichkeit für einen Folienvortrag zum Thema (Text und Folien). Die Folien werden im Aktionspaket präsentiert und der Preis genannt, bei Bestellung wird der Foliensatz separat in Rechnung gestellt

- Bestellschein für die kostenlose Anforderung von Broschüren und Give-aways (z. B. Probepackungen) von unterschiedlichen Firmen.

Das Aktionspaket ist für 20,- EUR, zzgl. 2,- EUR für Verpackung und Porto, erhältlich und wird im Februar 2016 verschickt. Alle Medien können in größerer Stückzahl nachbestellt werden. Wenn Sie schon jetzt wissen, dass Sie das Aktionspaket bestellen möchten, dann füllen Sie bitte den beiliegenden Bestellschein aus und senden diesen an die VFED-Geschäftsstelle.

Wir laden Sie ein, zusammen mit uns aktiv zu werden, um den Tag der gesunden Ernährung 2016 erfolgreich zu gestalten. Bei Fragen kann die Geschäftsstelle des VFED telefonisch unter 0241507300 oder per E-Mail (info@vfed.de) kontaktiert werden.

Wir freuen uns auf Ihre Teilnahme und wünschen Ihnen viel Spaß bei der Vorbereitung!

\section{Online}

http://dx.doi.org/10.1055/s-0035-1558487

\section{Quellen}

1 Statistisches Bundesamt

2 Deutsche Krebshilfe, Deutsche Krebsgesellschaft, Hrsg. Gesunden Appetit! Vielseitig essen - gesund leben [Präventionsratgeber]. http://www.krebshilfe.de/fileadmin/Inhalte/ Downloads/PDFs/Praeventionsratgeber/402_0034.pdf

3 Krebsinformationsdienst des Deutschen Krebsforschungszentrums. Ernährung: Einfluss auf das Krebsrisiko. https://www. krebsinformationsdienst.de/vorbeugung/ risiken/ernaehrung-praevention-index.php

4 Leitzmann C. Krebsprävention durch Ernährung. Ernährung und Medizin 2014; 29 : 55-58

5 Deutsche Krebsgesellschaft. 12 einfache Regeln schützen vor Krebs. http://www. krebsgesellschaft.de/onko-internetportal/ basis-informationen-krebs/vorsorge-undfrueherkennung/id-12-einfache-regelnschuetzen-vor-krebs.html\#ernaehrung
VFED e.V.

Eupener Str. 126

52066 Aachen

info@vfed.de

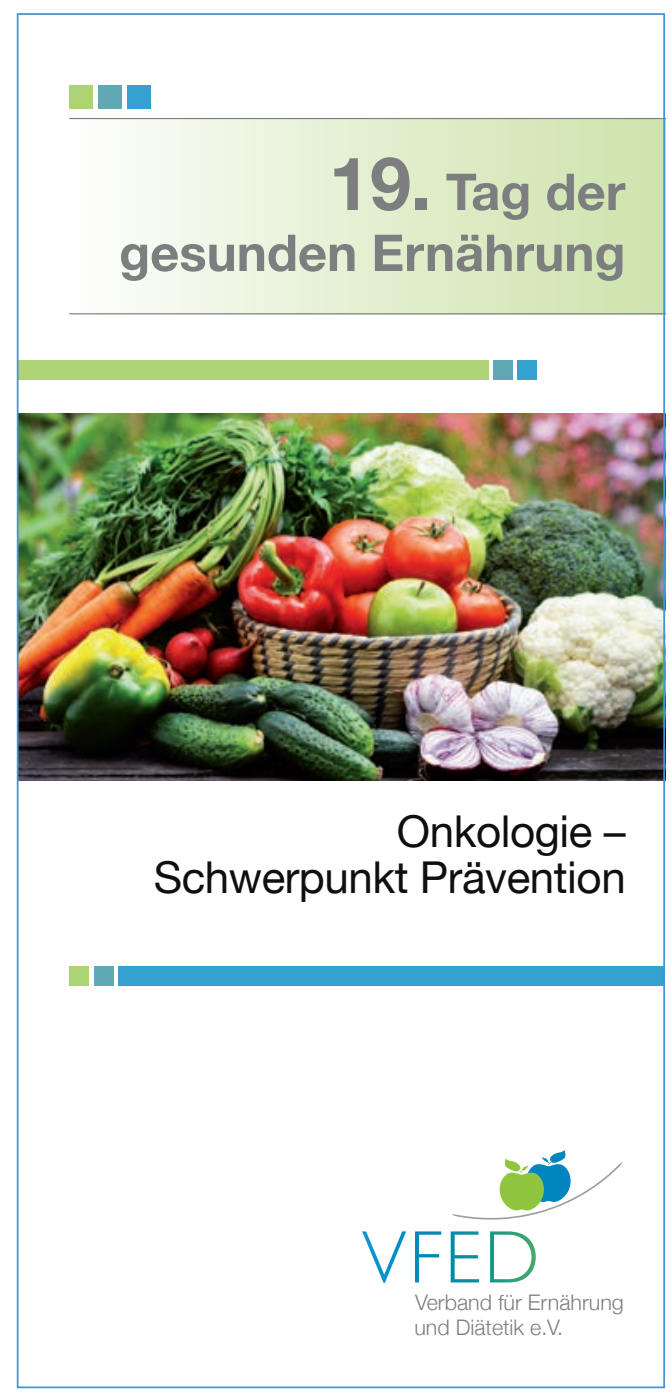




\section{Rückmeldung}

Ja, ich bin damit einverstanden, dass meine Anschrift an Institutionen, Firmen und Kolleg(inn)en weitergegeben wird, wenn diese an einer gemeinsamen Aktion mit mir interessiert sind.

Ja, ich möchte am Tag der gesunden Ernährung teilnehmen und bestelle das Aktionspaket.

\section{Bestellschein}

\begin{tabular}{|l|l|r|r|}
\hline $\begin{array}{c}\text { Anzahl } \\
\text { (bitte eintragen) }\end{array}$ & \multicolumn{1}{|c|}{ Titel } & Einzelpreis in EUR & Gesamtpreis in EUR \\
\hline & $\begin{array}{l}\text { Aktionspaket zum 19. Tag der gesunden } \\
\text { Ernährung Onkologie }\end{array}$ & 20,00 & \\
\hline & zzgl. 2,00 EUR für Verpackung und Versand & 2,00 & 2,00 \\
\hline & & Gesamtpreis & \\
\hline
\end{tabular}

Ort, Datum

Unterschrift

Der Preis gilt nur für Sendungen, die innerhalb Deutschlands verschickt werden. Den Preis für den Versand nach Österreich und in die Schweiz erfragen Sie bitte in der Geschäftsstelle des VFED e. V. (Telefon 0241507300 ).

Die Materialien werden geschickt an (Bitte in Druckbuchstaben schreiben! Danke):

Name

Straße, Hausnummer

Firma

(nur, wenn die Post an die Firmenanschrift geschickt werden soll!)

\section{Vorname}

PLZ, Ort

E-Mail

Datum, Unterschrift

\section{Einzugsermächtigung}

Hiermit ermächtige ich den Verband für Ernährung und Diätetik e. V. den Beitrag von 20,00 EUR, zuzüglich 2,00 EUR für Verpackung und Versand, für das Aktionspaket von meinem unten genannten Konto einzuziehen. 